\title{
Intervención sistémica con familias: de la linealidad a la circularidad ${ }^{*}$
}

DOI: https://doi.org/10.18046/recs.i28.2629

\author{
Systemic Intervention in Families: \\ From Linearity to Circularity \\ Intervenção sistêmica com famílias: \\ da linearidade à circularidade
}

\author{
Martha Luz Páez-Cala**
}

Universidad de Manizales (Manizales, Colombia)

\begin{abstract}
* El presente artículo expone, aplicado al campo de familia, un proceso investigativo realizado en la $\mathrm{V}$ cohorte de la Especialización en Psicoterapia y Consultoría Sistémica de la Universidad de Manizales, liderado por la autora en calidad de gestora del proyecto investigativo, investigadora principal y asesora. La investigación se realizó con algunos estudiantes del posgrado, los psicólogos Erika Lorena Arango Aristizábal, Leonardo Fabio Giraldo Marín, Lorena Pamplona Giraldo y Camila Zapata Quintero. Artículo de investigación recibido el 11.12.2017 y aceptado el 24.01.2018.

** Profesora asociada, psicóloga, especialista y magíster en Estudios de Familia y Desarrollo; excoordinadora, docente y asesora de prácticas e investigación de la Especialización en Psicoterapia y Consultoría Sistémica, del programa de Psicología, Facultad de Ciencias Sociales y Humanas, Universidad de Manizales. Correos electrónicos: mpaez@umanizales. edu.co, marthapaez315@yahoo.es ORCID: https://orcid.org/oooo-ooo1-7572-89oX
\end{abstract}




\section{Cómo citar/How to cite}

Páez-Cala, Martha Luz (2019). Intervención sistémica con familias: de la linealidad a la circularidad. Revista CS, 28, 207-227. https://doi.org/10.18046/recs.i28.2629 
Resumen

Abstract

Resumo

La presente es una investigación aplicada efectuada en un contexto formativo de orientación sistémica en posgrado, que buscó identificar algunos factores que propician, a terapeutas y consultores, tanto docentes como estudiantes en formación, el tránsito de un pensamiento lineal a un pensamiento circular, característico del quehacer sistémico, que opera mediante bucles de retroalimentación para potenciar el cambio. Metodología: se realizó mediante grupos focales y entrevistas a profundidad, en escenarios conversacionales reflexivos. Participantes: tres docentes orientadoras de seminarios teórico-prácticos y siete estudiantes de la Especialización en Psicoterapia y Consultoría Sistémica de la Universidad de Manizales. Resultados: se identificaron tres novedades en las experiencias de los psicólogos sistémicamente orientados: el pensamiento lineal y el circular no se vislumbran aislados ni excluyentes, puede transitarse de uno a otro; ello se constituye en una aventura propiciada por la necesidad de articular los dilemas humanos en un contexto de responsabilidad relacional, que implica la autorreferencia del interventor. Los participantes coinciden en factores básicos y relevan la transversalización de la esfera cognitiva, emocional y la pragmática en dicho tránsito, además, resaltan un movimiento novedoso al momento de sumergirse en lógicas de carácter circular.

\section{PALABRAS CLAVE:}

pensamiento sistémico, causalidad lineal, causalidad circular, reflexividad, formación sistémica

This is an applied research carried out in a formative context of systemic orientation in postgraduate studies. The objective was to identify some factors that favor the transition from a linear thinking to a circular one characteristic of the systemic task, which operates through feedback loops to enhance change, in therapists and consultants, both teachers and students in training. Methodology: It was carried out through focus groups and indepth interviews in reflective conversational settings. Participants: three lecturers of theoretical-practical seminars and seven students of the Specialization in Psychotherapy 
and Systemic Consultancy of the University of Manizales. Results: Three novelties were identified in the experiences of systemically oriented psychologists: linear and circular thinkings do not appear isolated or exclusive, they can be transposed from one to another. This constitutes an adventure facilitated by the need to articulate human dilemmas in a context of relational responsibility, which implies the self-reference of the intervener. Participants agree on basic factors and relieve the mainstreaming from the cognitive, emotional, and pragmatic sphere in this transit, also, highlight an innovative movement at the time of immersing in logics of circular nature.

\section{KEYWORDS:}

Systemic Thinking, Linear Causality, Circular Causality, Reflexivity, Systemic Formation

Está é uma pesquisa aplicada realizada em um contexto de formação de orientação sistêmica na pós-graduação, que procurou identificar os fatores que levam, terapeutas e consultores, professores e alunos em formação, o trânsito de um pensamento linear a um pensamento circular, característico de trabalho sistêmico, que opera através de ciclos de feedback para melhorar a mudança. Metodologia: foi realizada por meio de grupos focais e entrevistas em profundidade, em contextos conversacionais reflexivos. Participantes: três palestrantes de seminários teórico-práticos e sete alunos da pós-graduação em Psicoterapia e Consultoria Sistêmica da Universidade de Manizales. Resultados: três novidades foram identificadas nas experiências de psicólogos sistemicamente orientados: pensamento linear e circular não são vistos como isolados ou exclusivos, podem ser transpostos de um para outro; isto constitui uma aventura propiciada pela necessidade de articular dilemas humanos em um contexto de responsabilidade relacional, o que implica a auto-referência do interventor. Os participantes concordam em fatores básicos e modificam a transversalização da esfera cognitiva, emocional e pragmática em tais transito, também destacam um novo movimento ao momento de mergulhar em lógicas de caráter circular.

\section{PALAVRAS-CHAVE:}

pensamento sistêmico, causalidade linear, causalidade circular, reflexividade, formação sistêmica 


\section{Introducción}

El pensamiento sistémico, integrador por excelencia, alude a la percepción del mundo y su comprensión, en términos de totalidades y no de partes aisladas e inconexas. Se le califica como complejo dada la comprensión de sistemas abiertos, autopoiéticos, autorreferentes y dinámicos, en constante interacción y conexión entre los elementos integrantes del sistema como totalidad, y este, a su vez, con los sistemas del entorno. Esta perspectiva se centra en la noción de interacción, y postula al sistema como un conjunto de elementos que se relacionan e interactúan en forma dinámica e interdependiente (Bertalanffy, 1976; Watzlawick; Beavin; Jackson, 2011). Presuponer la existencia de una interdependencia entre las partes, lleva a la posibilidad de un cambio a través de la reversibilidad de la relación. Desde esta conceptualización, es posible imaginar infinidad de sistemas.

Dicha perspectiva, interaccional por excelencia, es muy pertinente cuando se abordan parejas, familias, comunidades y, en general, grupos sociales. Más allá de una concepción intrapsíquica, individual, característica de la psicología tradicional, aquella focaliza los procesos de interacción desde una visión de sistemas que interactúan y se retroalimentan, en un circuito de causalidad circular, desde una cibernética de segundo orden; de manera que un aspecto importante en la formación de interventores sistémicos tiene que ver con el tránsito de un pensamiento lineal a una perspectiva de causalidad circular.

En esta epistemología sistémica compleja, la noción de circularidad es clave, y los conceptos salud-enfermedad no son aislados, dado que el mundo se concibe como un complejo "entramado de relaciones", y se releva la importancia de considerar los vínculos que establecen las personas con los sistemas con quienes interactúan y, entre ellos, de manera relevante, la familia (Cohen, 2009: 70). La causalidad lineal, característica del pensamiento occidental, genera descontento en el terreno de la salud mental. En esta causalidad, el locus del funcionamiento deficiente se ubica en la persona, y la etiología se conecta con factores genéticos, bioquímicos o relacionados con el desarrollo intrapsíquico. Tradicionalmente, está representada en el modelo explicativo médico, que atribuye las disfunciones a factores biológicos, como causas de la enfermedad mental; y en el modelo psicodinámico, donde se atribuyen los síntomas a conflictos pasados, de la infancia, que han sido reprimidos.

Pero cuando se observa al individuo en su contexto, se vislumbra una red interaccional donde la persona sintomática es solo una pieza del contexto relacional recurrente. Inicialmente, fue Gregory Bateson (1979) quien profundizó en esta epistemología circular, al focalizar la interacción dinámica de los sistemas, su unidad y organización (Bertalanffy, 1976). Esto implica un tránsito de un pensamiento 
analítico, lineal, a un pensamiento integrativo, intuitivo, holístico, no lineal. La causalidad lineal es un modo de causalidad simple, que explica un efecto por una causa. La ecología profunda es un movimiento filosófico que considera la integración de la humanidad con el entorno que la rodea, en un encuentro armónico en su interacción; e incluye la percepción que tiene el ser humano del contexto en el que está inmerso. El objetivo de este movimiento es profundizar en la raíz de los dilemas humanos, involucrando al medio y a las personas, para realizar cambios profundos.

La epistemología sistémica se nutre de diversas fuentes, entre ellas, y de manera relevante, la teoría general de los sistemas (Bertalanffy, 1976), la epistemología cibernética (Wiener, 1988) y la teoría de la comunicación humana (Watzlawick et al., 2011). En primer lugar, la teoría general de los sistemas, de Bertalanffy (1976), enfatiza la "totalidad" y no la "sumatividad", y postula que cualquier cambio en un integrante del sistema afectará a los demás. De esta manera, las pautas de funcionamiento del sistema no son reducibles a la suma de sus elementos constituyentes. En este sentido, diversas causas pueden generar un mismo efecto, dada la constante circularidad e interconexión entre los integrantes de un sistema.

Por su parte, la cibernética de Wiener (1988) retoma el concepto de "homeostasis", según el cual a partir de la retroalimentación se tiende al mantenimiento de la organización del sistema, pues cualquier conducta de un integrante de un sistema se transforma en información para los demás. La epistemología cibernética se focaliza en la organización circular, no lineal, y en la pauta y, además, posibilita pasar de la ciencia de la información y el control a la ciencia de la comprensión.

La teoría de la comunicación, como tercera gran fuente de desarrollo teórico, tiene el siguiente axioma básico: "es imposible no comunicar", de manera que todo comportamiento es mensaje, comunicación, incluso el silencio, la mirada o la indiferencia. En el segundo axioma que establecen Watzlawick et al., (2011), destacan la diferenciación que debe establecerse en cuanto al contenido, la semántica y el aspecto relacional de toda comunicación; y en el tercero, plantean que toda definición de interacción está condicionada por la puntuación de una secuencia de hechos. A continuación, establecen que la comunicación humana implica una modalidad digital-lo que se dice- $\mathrm{y}$ otra analógica -cómo se dice-, y finalmente postulan que toda relación es simétrica o complementaria, según el criterio de igualdad o diferencia entre los participantes.

\section{Referentes conceptuales}

Se establece a continuación un horizonte conceptual que, de manera ágil y breve, sitúa en el sendero teórico recorrido por la ciencia para acceder a esta visión integral 
y compleja. Ello con el propósito de clarificar, desde este recorrido histórico, cómo se ubican los conceptos de causalidad lineal y circular, conceptos ejes del presente proceso investigativo, en cuanto a la manera como terapeutas y consultores sistémicos, en formación posgradual, acceden e interiorizan el tránsito desde la visión lineal para acceder a esta visión recursiva, compleja, al evaluar e intervenir dilemas humanos y familiares. A la vez, el recorrido ilustra la relevancia de este tránsito en una formación desde dicho paradigma. Posteriormente, se realiza un breve panorama acerca de la aplicación específica al campo de familia.

La causalidad circular supone un cambio epistemológico por el cual todos los elementos influyen sobre los demás y, a su vez, son influidos por estos. Se denomina retroalimentación a este intercambio circular de información, de forma que cada miembro adopta un comportamiento que influencia a los otros. El pensamiento sistémico es circular, en constante transformación mediante bucles de retroalimentación, bajo el postulado de que, si las partes cambian, por ende, también el sistema se transformará; esta retroalimentación posibilita un pensamiento en círculos (O'Connor; McDermott, 1998).

Bateson (1991) diferencia los objetos físicos, en los que se presenta una causalidad lineal y se ejerce una fuerza unidireccional sobre las cosas, de las formas vivas, donde, además de considerar la fuerza, se torna relevante la información y las relaciones. Ello trasciende la perspectiva lineal causa-objeto, para requerir un lenguaje recursivo, que propicie una comprensión integral acerca de las acciones, las interacciones y su influencia recíproca, que incluye, además, al contexto y muchos otros actores allí implicados. Es decir, se requiere de una epistemología circular.

El énfasis sobre las partes se asocia a visiones mecanicistas y reduccionistas, mientras el énfasis sobre el todo se asocia a visiones holísticas, ecológicas y organicistas. Se da el tránsito de una visión de las partes independientes a una visión interdependiente, donde emerge una observación de primer orden (lineal) y de segundo orden (circular), entendida esta última como una observación de la realidad social conformada por sujetos activos, quienes, a su vez, también observan, describen e interpretan, y que, además, asigna valor a cómo esto incide en la construcción de su propia realidad (Carrasco, 2016).

El pensamiento sistémico emerge en la primera mitad del siglo XX, con influencia de la biología, que conceptualiza a los organismos vivos como totalidades integradas y no separadas: de esta manera se pasa de una perspectiva de las partes a una perspectiva del todo. Posteriormente, se nutre de la psicología de la Gestalt y la ciencia ecológica, cuya filosofía transversaliza el cambio paradigmático de lo lineal a lo circular, como lo establece Capra (2011). Este autor concibe la ecología profunda como un nuevo paradigma que posibilita una comprensión holística del mundo, dado que 
visualiza las partes integradas en un todo, se les asigna un mismo valor a lo humano y al entorno, con una conexión entre ambos que permite el crecimiento mutuo.

Hacia la década de los ochenta, diversos terapeutas sistémicos (Hoffman, 1993; Sluzki, 1996; 1998) adoptaron, paulatinamente, una perspectiva constructivista, la cual postula un tránsito significativo: de las secuencias de conducta a la co-creación del significado. Se pasa del énfasis en la circularidad al énfasis en los significados compartidos de esas conductas (Feixas; Neimeyer, 1991), y se postula que el sujeto (observador) construye de forma activa su interpretación del mundo externo, de manera tal que coexisten diversos significados; lo cual dista del postulado ilusorio que propende por un conocimiento "verdadero" (Watzlawick, 1988). El constructivismo se constituye en una reflexión epistemológica que implica diversidad de intervenciones sistémicas (Neimeyer; Mahoney, 1998).

Lo anterior lleva a Lynn Hoffman a plantear la progresión desde el movimiento sistémico hacia uno sistémico constructivista (Hoffman, 1993). Si la teoría general de los sistemas tiene su mayor y más conocido campo de aplicación en la terapia familiar, los fundamentos e implicaciones filosóficas y epistemológicas del paradigma sistémico complejo tienen un carácter transdisciplinar, pues este paradigma emergente ha recibido el aporte de diversas ciencias, teorías, disciplinas, tecnologías e, incluso, de creaciones artísticas y literarias.

Desde una perspectiva de complejidad, se aspira al conocimiento de lo diverso y lo particular, a diferencia de una postura científica mecanicista que aspira acceder al conocimiento de lo universal. El paradigma de la complejidad, característico de la posmodernidad, implica una perspectiva abierta y sin leyes abarcadoras. Como humanos habitamos un determinado contexto cambiante y en movimiento, que implica novedad en los procesos investigativos/interventivos, y que condiciona cultural y socialmente, a la vez que provee de un lenguaje y un saber necesarios para deliberar con autonomía.

Por ejemplo, los planteamientos que surgen del constructivismo y, en especial, del construccionismo, en torno al afrontamiento generativo de crisis y conflictos en diversos contextos, se constituyen en un nuevo paradigma en comunicación y, particularmente, en la teorización acerca del cambio y cómo generarlo (Fried, 2000; 2010). La formación sistémica, constructivista y construccionista, bajo un paradigma de complejidad, suministra bases conceptuales para comprender, conceptualizar, intervenir e investigar los fenómenos humanos y sociales, a partir de comprensiones inter y transdisciplinarias; además de incitar lecturas generativas, contextuales, que propenden por abordar de manera integral los procesos de cambio y desarrollo social, bien sea a nivel familiar, comunitario, organizacional y demás contextos de intervención. 


\section{Perspectiva sistémica y familia}

En este campo, el modelo sistémico toma auge inicialmente hacia los años cincuenta, en el terreno de la terapia familiar (Feixas; Muñoz; Compañ; Montesano, 2016), pero su aplicación ha llegado a otros ámbitos, como es la intervención con familias, el bienestar familiar y social (Benavent, 2015; Gómez; Hincapié; Montoya; Moreno; Ramírez, 2007), la asistencia social, las redes sociales y en salud (Machin, 2010; Sluzky, 1996), el ámbito comunitario, escolar y organizacional (Medina; Agulló; Castro; Calderón; Eguiluz, 2009). El foco de acción son los procesos de interacción, más allá de la persona o de cada uno de los integrantes de un sistema familiar y social. Por ello, se considera un modelo interaccional por excelencia, que busca comprender la complejidad de los fenómenos interrelacionados que se presentan en un sistema dado.

Un sistema es un conjunto de elementos interdependientes que se relacionan entre sí, lo cual enfatiza la interacción y la relación; de esta manera, es posible deducir que un fenómeno se puede comprender cuando se amplía el foco de atención, es decir, cuando se reconoce el todo, dando relevancia al contexto, y no de manera aislada. Desde esta perspectiva, se observan y conceptúan las relaciones y las conductas por medio de la comunicación, para pensar sistémicamente en términos de conectividad, relaciones y contextos. Hall y Fagen (1956, como se citó en García; Musitu, 1993) exponen una definición actual de sistemas, al postular que se considera como sistema un conjunto de objetos y de relaciones entre ellos y entre sus atributos, donde los objetos son los componentes o partes del sistema, y los atributos son las propiedades de los objetos, cuyas relaciones se encargan de la unidad del sistema.

El paradigma sistémico se interesa en las posibles modificaciones de los sistemas de relaciones, plantea el paso del individuo al sistema, a lo interaccional. No se centra en una persona, familia o grupo "identificado", sino en las formas de organización del sistema en el cual se está inmerso. Desde este paradigma de complejidad y causalidad circular, en contraposición con uno de causalidad lineal, el trabajo con familias, en la variedad de niveles de intervención, hace referencia a la epistemología que orienta el análisis y abordaje de las diversas circunstancias. Como se afirmó, el foco no se centra en un sistema familiar específico, sino en el contexto, una visión ecosistémica de las situaciones y, por ende, de las familias. Al centrarse en la interacción como elemento de trabajo y comunicación, lo fundamental no es el "por qué", sino el "para qué" y el "cómo" se interactúa; la evaluación e intervención se focaliza en la estructuración, organización y funcionalidad familiar.

Bertalanffy (1976) formula el término "teoría general de los sistemas", como un mecanismo de integración entre las ciencias naturales y las sociales, que tiene como principio básico la totalidad orgánica. Se trata de una teoría que surge como respuesta 
al agotarse los enfoques analítico-reduccionistas, ya no aplicables, al igual que el principio mecánico causal que enunciaban, en sintonía con una visión inorgánica del mundo. Sus orígenes se ubican en los sistemas naturales -organismos-y sistemas mecánicos -máquinas-, aunque traslada su aplicación a los fenómenos culturales, sociales, familiares y humanos.

$\mathrm{El}$ concebir los fenómenos como sistemas organizados que se configuran en la relación con un contexto, se da un salto cuántico en la explicación de la naturaleza de los sistemas vivientes. Capra (2011) afirma que "en el planteamiento sistémico las propiedades de las partes sólo se pueden comprender desde la organización del conjunto, por lo tanto, el pensamiento sistémico no se concentra en los componentes básicos, sino en los principios esenciales de organización" (49).

Este tránsito de una perspectiva lineal a una circular surge de la insatisfacción con focalizarse solo en la apreciación lineal, y promueve una visión de mundo y su naturaleza ecológica desde una metapostura, más integrativa. Dicho tránsito se constituye en un momento crítico, a la manera de "crisis existencial para el dominio científico lineal", pero, igualmente, se constituye en oportunidad para efectuar un mayor acercamiento a la comprensión de la incertidumbre, al igual que a la complejidad de los sistemas vivientes y del entorno, entre ellos a la concepción de las familias, a su diversidad y especificidad. Retomando a Martínez Miguelez (1997), la “ontología sistémica" que exige y va en concordancia con una metodología interdisciplinaria implica un cambio radical en la conceptualización de los diversos fenómenos; es decir, las acciones humanas ya no se observan de manera aislada, sino en función de un todo, de un contexto, desde la óptica de lo que para un determinado momento se constituya como contexto focal.

\section{Agente de acompañamiento con familias}

La intervención familiar, desde esta perspectiva, incluye no solo al sistema familiar, sino también al sistema o equipo que lo interviene. En este escrito se asume la intervención como un encuentro basado en una co-construcción, bien sea a nivel de procesos de orientación, educativos, de empoderamiento, investigativos e incluso terapéuticos. Se habla desde una cibernética de segundo orden, o cibernética de los sistemas observantes, ya no de la cibernética de los sistemas observados, como se asume en la cibernética de primer orden. El observador forma parte de la realidad que observa, y ya no es el organizador quien genera cambios en la realidad con la cual interactúa, sino que se constituye en parte activa del fenómeno que observa, que interviene, que investiga.

Se gesta un proceso interaccional, donde las observaciones son relativas según su perspectiva. Al observar, se incide sobre el objeto observado y se excluye toda 
expectativa de predicción por parte del observador; se da paso a la incertidumbre e indeterminación (Ceberio; Watzlawick, 1997). En la epistemología de los sistemas vivientes, se reintroduce al observador, sin pretensión de neutralidad y objetividad. La cibernética de segundo orden lleva a la observación del observador, el foco que se anexa es el observador observando su propia observación.

Al intervenir con sistemas familiares desde esta epistemología, en un encuentro que busque generar diversos efectos (empoderamiento, afrontamiento, procesos de reflexión, superación y cambio), son esenciales el vínculo afectivo y la convivencia. Esto lleva a recordar el término de tutor de resiliencia (Cyrulnik, 2003), concepto que sugiere ciertas características que debe poseer un agente de intervención o un acompañante de sistemas familiares, bien sea a nivel de orientación, educativo, de promoción de resiliencia, atención directa en situaciones críticas o, incluso, un terapeuta. Lo esencial es la capacidad para estimular un renacer del desarrollo integral luego de una situación difícil (Cyrulnik, 2001).

El encuentro con un sistema familiar que amerita algún grado de acompañamiento o intervención puede considerarse como un contexto co-creado por todos los participantes, que propicia identificar las voces provenientes de diversos actores, personajes y contextos, que reflejan acciones, significados y reflexiones inmersas en las historias compartidas (Garciandia; Samper, 2004). "Las narrativas son perspectivas inmersas al interior del ser humano, quien se vincula con el relato desde su propia autobiografía" (Páez, 2016:346). Este encuentro, como contexto que busca generar un cambio, puede definirse "como un espacio conversacional propio, que implica construcción de narrativas terapéuticas en las que al entretejer las historias, se integran las vivencias significativas y aspectos distintivos del terapeuta y de sus consultantes" (Páez, 2016:346).

\section{Propósito de indagación}

En el paradigma sistémico, toma fuerza la experiencia del interventor, sujeto observador y participante activo en la construcción del conocimiento y del cambio. De acuerdo con Eduardo Carrasco (2016), la circularidad implica pensar lo otro como si formara parte de la esencia de sí mismo y, a su vez, de lo otro; conformándose, entonces, una nueva unidad: el otro y el sí mismo. Según este autor, el pensamiento circular alude "al pensamiento que se incluye dentro de lo pensado, en el mismo momento en que lo piensa" (Carrasco, 2016: 39). El pensamiento sistémico es circular, en constante transformación mediante bucles de retroalimentación, bajo el postulado de que, si las partes cambian, por ende, también el sistema se transformará; esta retroalimentación posibilita un pensamiento en círculos (O'Connor; McDermott, 1998; Sandoval, 2003). 
La experiencia docente, tanto en pregrado como en posgrado, permite identificar que, en este proceso de contextualización, de abordar fenómenos complejos, interdependientes, los estudiantes en formación vislumbran confusiones que asumen como excluyentes, en especial cuando previamente se han focalizado de manera exclusiva en la causalidad lineal y en los fenómenos intrapsíquicos. Varios de ellos los asumen como principios que deben abandonar, generando confusiones en relación con los conceptos novedosos y relevantes que privilegian y asumen en sus cosmovisiones.

Los postulados sistémicos tienden a generar admiración y desconcierto en los participantes de estos procesos formativos, independiente del campo específico de intervención, bien sea desde las ciencias sociales o de la salud. De allí el interés en identificar algunos factores que propician a los terapeutas y consultores, tanto docentes como estudiantes en formación, el tránsito de un pensamiento lineal a un pensamiento circular.

\section{Metodología}

El componente metodológico se inscribe en un proceso de investigación cualitativo con elementos de segundo orden, a la manera de una observación sistémico-constructivista, donde quien observa se focaliza no solo en sus propias observaciones, sino también en otros observadores. Este observador de segundo orden, para Arnold (1998), al observar releva lo que sus observados no pueden distinguir. Implica una postura de metaobservación muy activa, además de hacerse responsable de sus reflexiones.

En el presente caso, el grupo de investigadores se insertó como actor activo que participaba en la indagación y en el tejido conceptual que surgió de la misma, aportando al proceso conversacional que estimula la reflexión, desde sus experiencias y significados que emergían en el encuentro. A partir de este proceso conversacional, se gestaron observaciones y desarrollos consensuales, que, según Jairo Estupiñán (2003), desde una posición de curiosidad, permiten identificar dos dominios: uno narrativo que focaliza el análisis del discurso y concepciones de mundo, y un dominio autorreferencial, reflexivo que focaliza las experiencias autopoiéticas de los sistemas humanos. A su vez, esta reflexividad propicia construcciones y un conocimiento de segundo orden (Estupiñán, 2003), que emerge en esta construcción colectiva y consensuada, en busca de elucidar comprensiones acerca de los diversos sentidos que surgen en los encuentros conversacionales sobre el tema investigar.

En este proceso recursivo de la reflexión, el observador participa activamente de lo observado, relaciona la información con su propia experiencia y lo vincula con lo que emerge (Molina, 2001). Más allá de ver, como lo hace el observador de primer 
orden, el de segundo orden además reflexiona, saca conclusiones y actúa en relación con su motivación original para indagar, y articular su experiencia a lo que surge de ese otro. De esta manera, se genera más información, novedosa, relacionada, y se constituye en un observador de sistemas complejos, dado que observa y opera en realidades que él mismo construye.

Se trata de sujetos activos, quienes además de observar la realidad, la describen e interpretan. Epistemológicamente, esta observación de segundo orden es reflexiva, dado que incluye al observador en la observación científica y en la conciencia acerca de que lo observado lo construye un observador (Mejía, 2002). Desde esta perspectiva, no es posible separar al investigador de las observaciones realizadas, ya que son sus intereses personales y profesionales los que permiten que la investigación se lleve a cabo (Bautista, 2011). La postura de los investigadores/interventores se genera a partir de su autorreferencia y permanente exploración del proceso personal durante la formación como terapeutas en el espacio del posgrado, se fomenta un diálogo reflexivo entre las observaciones planteadas y las posiciones personales, desde una postura respetuosa, espontánea.

\section{Diseño}

El proceso investigativo se efectuó en dos momentos: primero, mediante estrategias conversacionales, reflexivas, con tres docentes orientadores de seminarios teórico-prácticos, y estudiantes que cursaban la V cohorte de la Especialización en Psicoterapia y Consultoría Sistémica de la Universidad de Manizales. Posterior a ello, se analizaron, desde una perspectiva hermenéutica, los factores que inciden en el tránsito de un pensamiento lineal a un pensamiento circular, a partir del relato de las docentes y los estudiantes en formación, que participaron en el proceso investigativo. Con las docentes se realizaron entrevistas a profundidad, y con los estudiantes en formación se inició con un grupo focal, con el cual, a continuación, se efectuaron algunas entrevistas a profundidad, según lo establecido en este tipo de estrategias metodológicas (Sandoval, 1996).

\section{Participantes}

Tres psicólogas con formación posgradual en perspectiva sistémica, con edades comprendidas entre los 32 y 64 años, con experiencia docente y asistencial desde esta perspectiva, que eran docentes de seminarios teórico-prácticos del posgrado en mención. Además, se contó con la participación de siete estudiantes que cursaban la V Cohorte de la Especialización en Psicoterapia y Consultoría Sistémica, como se detalla a continuación: 
TABLA 1 Estudiantes participantes en la investigación

\begin{tabular}{|c|c|c|c|c|c|}
\hline Estudiante & Género & Edad & Estado civil & $\begin{array}{l}\text { Experiencia } \\
\text { laboral }\end{array}$ & $\begin{array}{c}\text { Contexto } \\
\text { laboral }\end{array}$ \\
\hline 1 & Masculino & 22 & Soltero & 1 año & Clínico jurídico \\
\hline 2 & Femenino & 23 & Soltera & 7 meses & Clínico educativo \\
\hline 3 & Femenino & 36 & Unión libre & 5 años & Organizacional \\
\hline 4 & Femenino & 42 & Casada & 15 años & Organizacional clínico \\
\hline 5 & Masculino & 25 & Soltero & 7 meses & Educativo \\
\hline 6 & Femenino & 25 & Soltera & 6 meses & Clínico social \\
\hline 7 & Femenino & 23 & Soltera & 4 meses & Clínico social \\
\hline
\end{tabular}

\section{Resultados}

Las categorías emergentes surgen del proceso reflexivo sobre las entrevistas con los integrantes de los grupos focales, conformados por los mencionados estudiantes y tres docentes de la misma especialización. Ellos aportaron reflexiones sobre su experiencia, como insumos para indagar acerca de la relación entre el pensamiento lineal y el circular, al igual que algunos factores que, según su experiencia formativa, inciden en dicho tránsito.

A partir de la indagación a profundidad, durante el diálogo y posterior a él, con los resultados que emergen de los procesos conversacionales, el grupo de investigadores reflexionaron acerca de sus propias experiencias. Sobre la base de la problematización focal propuesta, surgieron los tres planteamientos que se exponen a continuación.

\section{El pensamiento lineal y el pensamiento circular no son vistos de manera dicotómica}

Se comprendió que, si bien en el ámbito académico y literario, por momentos, surge una sensación de dicotomía entre el pensamiento lineal y el circular, parece ser que en la praxis profesional estas dos epistemes se integran, y la una no es excluyente de la otra. En palabras de un estudiante del posgrado, "el pensamiento circular es el pensamiento lineal en multiplicidad de veces" (Estudiante $n .^{\circ} 1$, comunicación personal). 
Se evidenció que los docentes y estudiantes, más allá de optar por un pensamiento taxativo y excluyente, eligieron velar por que sus referentes de intervención fueran coherentes y establecieran conexiones, teniendo en cuenta el sentido de su intervención y el contexto en el cual se realizaba.

\section{El tránsito del pensamiento lineal al pensamiento circular es una aventura que implica la autorreferencia}

Se reconoció, en la narrativa de los participantes del grupo focal, un aspecto que se tornó relevante cuando denotaron el tránsito del pensamiento lineal al pensamiento circular, y es, precisamente, la transversalización de la esfera cognitiva, la emocional y la pragmática en dicho tránsito. Si bien mencionaron que en la praxis no se excluían los dos tipos de pensamiento, a priori, bajo formación sistémica, sí daban cuenta de un movimiento novedoso al momento de sumergirse en lógicas de carácter circular.

En la esfera cognitiva, toma forma un tipo de razonamiento que describieron como flexible, espontáneo, incluyente y no taxativo. Esto último, según Zaldívar, Sosa y López (2006), es lo que posibilita el empleo de recursos cognitivos en la búsqueda de alternativas. Según los participantes, este tipo de razonar espontáneo facilita la apertura emocional, brinda la sensación de conexión y respeto por su sistema consultante, lo que -en palabras de ellos- pragmáticamente los dota de recursos inagotables cuando se amplía la naturaleza de los fenómenos que se observan.

En este orden de ideas, el tránsito implica un auto-observarse, posicionarse en una lógica reflexiva frente a las tendencias, intereses, sesgos, constructos mentales y actitudes, ya que pensar epistemológicamente la intervención implica, según la docente con mayor experiencia laboral, una "responsabilidad relacional", que conlleva comprender desde dónde se observan los fenómenos, en términos del referente conceptual, contextual, de historia de vida, y la intencionalidad subyacente. Y, en especial, qué es lo que se desea generar.

Según el relato de esta docente, el pensamiento circular brinda una gran riqueza informativa y lleva a asumir la responsabilidad de los propios actos. Esto lo torna muy incluyente, vinculante y hasta comprometedor, en cuanto a asumir la propia responsabilidad al realizar un acompañamiento. Ella lo denomina "responsabilidad relacional", aspecto que -según enuncia- es ineludible en los encuentros con familias desde una perspectiva sistémica, y el cual debe conducirse para que se asuma la responsabilidad del estilo cognitivo, actitudes, paradigmas, sesgos; implica observar desde dónde y de qué manera se participa en la interacción. En palabras de esta docente: 
El pensamiento circular es muy incluyente, vinculante y comprometedor, en la medida en que se focaliza la participación de los diferentes personajes en un hecho, se amplía el foco y se empieza a mirar lo que denomino responsabilidad relacional, que es algo que con frecuencia se diluye en el sentido en que se tiende a responsabilizar a otros de las situaciones. Esto no significa que se deba satanizar el pensamiento lineal, las preguntas lineales por ejemplo llevan a la construcción de preguntas circulares si nos movemos con experticia entre ambas miradas (Docente, comunicación personal).

Se trata de propiciar un encuentro generativo, propositivo, respetuoso y de coparticipación, centrado en los recursos. Es un contexto de calidez, sin culpabilizar, en pro de que los sistemas familiares se sientan comprendidos, acogidos, en un clima de contención, confianza, respeto por la diferencia, singularidad, seguridad, protección y apoyo.

\section{El tránsito del pensamiento lineal al pensamiento circular es propiciado por la necesidad de articular los dilemas humanos en un contexto relacional, donde se amplía el mapa en comparación con la lógica causa-efecto}

Se identificó que lo circular es una cualidad del observador, es un lente por el cual se observa la realidad de otra manera, más incluyente, totalitaria y más amplia. Igualmente, se estableció que no necesariamente hay que desarrollarla en los cursos y en la academia, ya que, como menciona un estudiante de la especialización, "la circularidad está implícita en la naturaleza, en las estrellas, en el mar, en las plantas, en las relaciones humanas, simplemente tenemos que cambiar el foco para poder impregnar nuestra retina de ella" (Estudiante n. ${ }^{\circ}$, comunicación personal).

El pensamiento circular propicia establecer conexiones. Según otra docente, en la práctica profesional "el pensamiento circular propicia comprender que los sujetos estamos inmersos en sistemas complejos, es decir, redes entre redes; ello hace que se valoren los motivos de consulta de manera situada, contextual, de acuerdo con las historias de vida familiares". Lo humano surge en las interacciones.

Se comprendió que este tránsito muchas veces es propiciado por la naturaleza de las relaciones, que conllevan la necesidad de comprender de qué manera los sujetos están inmersos en sistemas complejos, en redes. Se vislumbra que lo humano surge en las interacciones, que en los encuentros con otros se configuran las formas de entender y moverse en la realidad; de hecho, se advierte que existen múltiples formas de ver la realidad, a partir de las experiencias, sistemas de creencias y de los significados que se asignan a la vida. 


\section{Discusión}

A través de la presente investigación se logró comprender de qué manera, en la práctica, el pensamiento circular no excluye al pensamiento lineal, ya que, dependiendo de la experticia del interventor, estas dos lógicas de pensamiento se pueden integrar en beneficio del encuentro con los sistemas a quienes se les brinda algún tipo de acompañamiento. Igualmente, amplía la mirada frente a la naturaleza compleja de los fenómenos humanos.

La tendencia por dicotomizar está arraigada a una cultura que exige la diferenciación entre las características de la naturaleza humana, demandando, constantemente, una posición excluyente, no integradora, en una u otra mirada de las lógicas que construye el ser humano para comprender la realidad; esto trae como resultado el no concebir los matices y las diversas tonalidades que coexisten. Por tanto, en el presente ejercicio investigativo, la perspectiva integral e incluyente es una prioridad, aunque, si bien teóricamente en el tiempo hay una diferenciación de dichos paradigmas, en la práctica estas dos epistemes se integran para dar cuenta de los fenómenos a observar y comprender.

Otro resultado relevante es que, según los participantes, la formación sistémica genera innovaciones en la manera de observar la realidad, que implica ponerse unos lentes incluyentes y generativos para dar cuenta de ella. Toma relevancia el hecho de que este tránsito está transversalizado, inevitablemente, por el proceso autorreferencial de cada sujeto que lo realiza, ya que implica deliberar acerca de la forma como cada uno comprende y reflexiona en torno a sus paradigmas, constructos, actitudes, creencias y mitos culturales, para dar cuenta de la realidad que observa, lo que, sin duda, permea las actuaciones profesionales.

Luhmann (2005) diferencia tres niveles de observación: de primero, de segundo $\mathrm{y}$ de tercer orden. El observador de primer orden se vincula con las cosas sin mediación; es decir, no "observa su propia relación de observación", siendo este último aspecto característico del observador de segundo orden, quien, a su vez, lo es de primer orden, pero lo trasciende. Define entonces al observador de tercer orden como quien "observa al observador que observa". Esto recuerda lo planteado por Jesús Ibáñez (como se citó en Molina, 2001), en el sentido de que en el primer orden se piensan los objetos, mientras que en el segundo orden o pensamiento complejo de los sistemas observadores se piensa el pensamiento.

Esto se evidencia en el relato de los participantes, quienes afirmaron que el tránsito del pensamiento lineal al pensamiento circular siempre lleva implícita una metaobservación y un darse cuenta de la manera como cada uno interactúa con los otros, 
desde su ser. El sentido del acompañamiento con sistemas humanos, para el presente texto con familias, pareciera requerir de una reflexión consciente de las propias intencionalidades, en el encuentro con un otro. Reflexión que posibilita relacionamientos más generativos, respetuosos, incluyentes, y menos viciados por aquellos sesgos y prejuicios que no dan la oportunidad de expandir la esencia de lo humano.

De esta manera, es posible estructurar sistemas comprensivos a partir de la observación autorreferencial, organizada en forma recursiva. Ello implica tomar en consideración los factores multicausales y multidimensionales, contextuales, interaccionales, y los procesos organizativos alrededor de la cosmovisión de fenómenos complejos. Tener en cuenta la interacción e interdependencia mutua incluye, por supuesto, al agente de acompañamiento con familias; e implica, igualmente, un pensamiento en red, en términos de diversidad, unidad e identidad de un sistema familiar en particular. El agente de acompañamiento familiar es parte del proceso, e incide en el mismo, de allí el concepto de responsabilidad relacional.

Se concluye que la salud de los integrantes de un sistema se conecta, a su vez, con la salud del ecosistema. Existen fuerzas que generan equilibrio, tendencia a la homeostasis, pero también aquellas que desestabilizan, dada la predisposición y necesidad de adquirir grados superiores de desarrollo. En ello incide el lugar que se ocupa y que crea ciertas necesidades, bien sea de orden físico, social o psicológico, cuya satisfacción se vincula estrechamente con la relación salud-enfermedad. De aquí se deduce que los procesos salutogénicos implican un equilibrio entre la satisfacción de necesidades y una respuesta satisfactoria; donde el desequilibrio surge cuando la capacidad de respuesta es ineficiente.

\section{Conclusiones}

Sin duda, para las ciencias sociales, la revolución del pensamiento circular contiene la piedra angular para la comprensión de los fenómenos desde una posición más holística e incluyente, lo cual genera un pensamiento que tiene en cuenta la complejidad, lo diverso, lo interaccional, las pautas, las interconexiones, entre otros. Justamente, la actitud y postura que se requiere para asumir la complejidad y diversidad de los sistemas humanos en general y, para el presente texto, de los sistemas familiares.

Esta epistemología se aparta de la lógica cartesiana que planteaba que para comprender el todo era necesario fragmentar las partes. Postura característica de una epistemología lineal, que ha transversalizado el pensamiento de Occidente durante cientos de años, pero que, ante la magnitud y singularidad de las realidades humanas, parece que la lógica de objetividad y primer orden no coincide con las demandas de los dilemas humanos, de los sistemas familiares, en su complejidad ecosistémica. 
Abordar los sistemas familiares desde una perspectiva sistémica, implica dejar de lado las visiones totalitarias, excluyentes, fragmentarias y dicotómicas, para acceder a esta nueva aventura del saber. Implica, además, una responsabilidad relacional entre el agente de acompañamiento con familias y los sistemas familiares que interviene, en donde el observador, en su estilo característico de operar, que lo define, configura sus observaciones, comprensiones y conceptualizaciones. Y, en ellas, plasma parte de su historia, sus conceptualizaciones y epistemología.

Limitaciones: ninguna en especial.

\section{Referencias}

Arnold, Marcelo (1998). Recursos para la Investigación Sistémico/Constructivista. Cinta de Moebio, 3. Recuperado de http://www.redalyc.org/articulo.oa?id=10100305

Bateson, Gregory (1979). Mind and nature: A necessary unity. New York, NY: E. P. Dutton.

Bateson, Gregory (1991). Pasos hacia una ecología de la mente. Buenos Aires: Carlos Lohlé-Planeta.

Bautista, Nelly (2011). Proceso de la investigación cualitativa: epistemología, metodología y aplicaciones. Bogotá: Manual Moderno.

Benavent, Encarna (2015). La intervención con adolescentes y familias desde el enfoque sistémico. En El marco global de atención al menor (pp. 297-314), editado por José Navarro; Vicenta Mestre. Valencia: Tirant Humanidades.

Bertalanffy, Ludwing-von (1976). Teoría General de los Sistemas. Fundamentos, desarrollo, aplicaciones. Ciudad de México: FCE.

Capra, Fritjof (2011). La trama de la vida, una nueva perspectiva de los sistemas vivos. Barcelona: Anagrama.

Carrasco, Eduardo (2016). Notas sobre el pensamiento circular. Revista de Filosofia, 45(46), 37-54.

Ceberio, Marcelo; Watzlawick, Paul (1997). La construcción del Universo. Buenos Aires: Herder.

Cohen, Jorge (2009). Saludy enfermedad, una aproximación desde la Teoría Sistémica. Perspectivas psicológicas en salud. Montevideo: Psicolibros.

Cyrulnik, Boris (2001). La maravilla del dolor: el sentido de la resiliencia. Buenos Aires: Granica

Cyrulnik, Boris (2003). El murmullo de los fantasmas: volver a la vida después de un trauma. Barcelona: Gedisa. 
Estupiñán, Jairo (2003). Algunos principios orientadores en los procesos de investigación, intervención y formación de terapeutas y consultores de familia. Construcciones en psicología compleja, aportes y dilemas. Bogotá D. C.: Universidad Santo Tomás.

Feixas, Guillem; Neimeyer, Robert (1991). La perspectiva constructivista: un marco integrador para la psicoterapia. Boletín de Psicología, 30, 7-33.

Feixas, Guillem; Muñoz, Dámaris; Compañ, Victoria; Montesano, Adrián (2016). El modelo sistémico en la intervención familiar. Barcelona: Universidad de Barcelona.

Fried, Dora (comp.) (200o). Nuevos paradigmas en la resolución de conflictos. Perspectivas y prácticas. Montevideo: Granica.

Fried, Dora (2010). Perspectiva generativa en la gestión de conflictos sociales. Revista de Estudios Sociales, 36, 51-63.

García, Enrique; Musitu, Gonzalo (1993). El maltrato infantil: un análisis ecológico de los factores de riesgo. Madrid: Ministerio de Asuntos Sociales.

Garciandia, José; Samper, Jeannette (2004). El tejido de un nosotros: hilando nuevos significados entre terapeuta y consultante. Revista Colombiana de Psiquiatría, 33(3), 262-284.

Gómez, Claudia; Hincapié, Marcela; Montoya, María; Moreno, Claudia; Ramírez, Viviana (2007). Reflexionesy aportes de la consultoría en familia como estrategia para la construcción de relaciones democráticas en las familias (Trabajo de grado). Universidad de la Salle, División de Formación Avanzada, Especialización en Consultoría en Familia y Redes Sociales, Bogotá.

Hoffman, Lynn (1993). Exchanging Voices: A Collaborative Approach to Family Therapy. Londres: Karnac Books.

Luhmann, Niklas (2005). El arte de la sociedad. Ciudad de México: Herder.

Machin, Juan (2010). Modelo ECO2: Redes sociales, complejidad y sufrimiento social. Redes, 18(12), 305-325.

Martínez, Miguel (1997). El paradigma emergente. Hacia una nueva teoría de la racionalidad cientifica. Ciudad de México: Trillas.

Medina, Raúl; Agulló, Esteban; Castro, Remberto; Calderón, Rocío; Eguiluz, Luz (2009). Consultoría colaborativa. Intervención sistémica en redes y organizaciones. México: E-Libro Red Américas.

Mejía, Julio (20O2). Perspectiva de la investigación social de segundo orden. Cinta de Moebio, $14,200-225$.

Molina, Silvia (2001). La investigación de segundo orden en Ciencias Sociales y su potencial predictivo: el caso del proyecto de identidad y tolerancia. Revista Mexicana de Ciencias Políticas y Sociales, XLIV(183), 17-46. 
Neimeyer, Robert; Mahoney, Michael (eds.) (1998). Constructivism in Psychotherapy. Washington, DC: APA.

O'Connor, Joseph; McDermott, Ian (1998). Introducción al pensamiento sistémico. Recursos esenciales para la creatividad y la resolución de problemas. Buenos Aires: Ediciones Urano.

Páez-Cala, Martha-Luz (2016). Experiencias resilientes del terapeuta generadoras de cambio a través de expresiones plásticas. Archivos de Medicina, 16(2), 345-358. https://doi. org/10.30554/archmed.16.2.1834.2016

Sandoval, Carlos (1996). Investigación cualitativa. Bogotá: ICFES.

Sandoval, Humberto. (2003). El bucle: referencia y autorefrerencia en la construcción del conocimiento. En Construcciones en psicología compleja. Aportes y dilemas (pp. 9-12), editado por Jairo Estupiñán; Ángela Hernández; Milva Barragán; Diana Rodríguez; Mónica Polo et al. Bogotá: Universidad Santo Tomás.

Sluzky, Carlos (1996). La red social: frontera de la práctica sistémica. Barcelona: Gedisa.

Sluzky, Carlos (1998). Terapia familiar como construcción de realidades alternativas. Sistemas familiares, 1, 53-59.

Watzlawick, Paul (1988). La realidad inventada. Barcelona: Gedisa.

Watzlawick, Paul; Beavin, Janet; Jackson, Don (2011). Teoría de la comunicación humana. Interacciones, patologías y paradojas. Barcelona: Herder.

Wiener, Norbert (1988). Cibernética y sociedad (3. ${ }^{\mathrm{a}}$ ed.). Buenos Aires: Sudamericana.

Zaldívar, Miguel; Sosa, Yamilka; López, José (2006). Definición de la flexibilidad del pensamiento desde la enseñanza. Revista Iberoamericana de Educación, 37(4), 1-5. 